\title{
Epicardial cardiac cavernous Haemangioma-a case report
}

\author{
Mahmoud Yousef Ibrahim Abuharb®D, Xiao Ming Bian and Jian He*
}

\begin{abstract}
Background: Cardiac haemangiomas are exceptionally rare. They are usually solitary growths. Cardiac haemangiomas can be classified as capillary, cavernous, or arteriovenous in nature. They can occur in any chambers of the heart, but are predominantly found at the intramural or endocardial layers.

Case presentation: This is a rare case of a cardiac haemangioma located on the epicardium of a 52-year-old male patient. The patient complained of 1-year duration of chest tightness and shortness of breath. The haemangioma was removed successfully. For symptomatic lesions, surgical removal remains the preferred treatment.

Conclusion: The pathological diagnosis was primary cardiac cavernous haemangioma. In this case, the haemangioma was successfully resected with invasive surgery. No recurrence was detected on follow up.
\end{abstract}

Keywords: Heart neoplasms, Haemangioma, Mitral stenosis, Rheumatic heart

\section{Background}

Haemangiomas refer to the type of tumours of blood vessel cell types. They develop from vascular endothelial hyperplasia and are usually benign in nature. Cardiac haemangioma is a very rare type of benign heart tumour, accounting for only $2.8 \%$ of all primary cardiac tumours [1]. The autopsy detection rate ranged from as low as 0.0017 to $0.27 \%$ in a recent study [2]. The sites of origin of cardiac haemangioma can be from any of the three cardiac layers, either endocardium, myocardium, or epicardium. In some cases, cardiac haemangiomas can also be found across the whole pericardium [3]. Among all, the epicardium is the rarest site of origin for this tumour. In addition, in terms of the anatomic sites of origin, slightly more than one third $(36 \%)$ of the cardiac haemangiomas are found in the right ventricle, another one-third (34\%) were from the left ventricle. Approximately $23 \%$ of the cardiac haemangiomas are located in the right atrium (23\%) and only very few (7\%) cases are found in the left atrium. In terms of vascular classification, there are 3 types of cardiac haemangiomas; namely

\footnotetext{
*Correspondence: 2258356549@qq.com

All authors confirmed that this manuscript has not been previously published and is not currently under consideration by any other journal. Additionally, all of the authors have approved the contents of this paper and have agreed to the policies BMC Cardiovascular Disorders.

The first affiliated hospital of Dalian Medical University, Lianhe Avenue, Dalian, China
}

cavernous tumour with large, thin-walled vascular spaces, arteriovascular tumour which is an unorganized malformation of arteries and veins, and lastly tumour with hypervascular capillaries [4].

The growth mechanism of cardiac haemangioma is not fully understood. It can present simultaneously with other heart diseases, resulting in diagnostic dilemma in certain cases. In cases of primary pericardial neoplasms, patients often present with various types of localized symptoms, such as exertional dyspnoea, chest pain, cough, palpitations; and systemic symptoms including fatigue, night sweats, fever, and facial or lower limb oedema. Among all, the most commonly reported symptom by the patients is exercise intolerance [5]. Nevertheless, it has also been recently reported in the literature on how a primary cardiac haemangioma was detected in a completely asymptomatic patient who presented for an unrelated illness [6].

In view of the wide variety in the symptom presentation, imaging modality is an important component in the investigation of primary cardiac haemangioma. Initial imaging approach such as chest radiography (CXR) or transthoracic echocardiography have limited value. On the other hand, magnetic resonance imaging (MRI) and computed tomography $(\mathrm{CT})$ are more useful as these cross-sectional imaging modalities allow further characterization to the condition, and may, in some cases, provide diagnostic findings. With respect to treatment option, cardiac 
haemangiomas are not always amenable to surgery. Successful resection can be performed only if the tumour is well circumscribed and small. In this case report, we outline the case of an adult male who presented with epicardial cardiac haemangioma.

\section{Case presentation}

A 52-year-old-male patient presented to the hospital on 4th of January 2018 with a 1-year duration of chest tightness and shortness of breath. The symptoms had been worsening for the past 1 month. Physical examination showed BP120/70 mmHg, HR 72/min. Electrocardiography (ECG) showed a heart rate of $72 / \mathrm{min}$, sinus rhythm and an incomplete right bundle branch block. Echocardiography $(\mathrm{ECHO})$ of the heart revealed that the left atrium was $56 \times 72 \mathrm{~mm}$, the right atrium was $42 \times 56 \mathrm{~mm}$, the left ventricle was $42 \mathrm{~mm}$, the right ventricle was $19 \mathrm{~mm}$. Left ventricular ejection fraction (LVEF) was 56\%. Mitral valve disease with valve leaflet thickening, left atrial enlargement, thrombosis over the left atrial anterior wall and left atrial appendage was also noted. Arterial blood gas analysis was normal. Computed tomography angiography (CTA) with iodine based contrast medium was performed. It revealed a 3 $\times 2 \mathrm{~cm}$ mass on the epicardium, located just over the anterior wall of the right ventricular outflow tract (Figs. 1, 2 and 3). All these findings were consistent with the diagnosis of cardiac haemangioma. Patient was also revealed to have underlying rheumatic heart disease, specifically mitral stenosis with regurgitation during the investigation.
On day 5 of admission, patient underwent open surgery for tumour resection and mitral valve replacement. Median incision was performed, followed by cannulation of aorta, superior, and inferior vena cava. Cardiopulmonary bypass was established. Under moderate temperature and myocardial protection with cold blood cardioplegia, myocardial anterograde and retrograde cardioplegia perfusion through the aortic root and coronary sinus were established. A heart-shaped mass was visible on the surface of the anterior wall of the right ventricle. It was about $3 \times 2$ $\mathrm{cm}$ in size. The capsule was intact with a dark red appearance and tough texture. There was no adhesion to the surrounding tissue, and also no connection of blood vessels between the tumour and the heart, the haemangioma was nourished by general superfusion of blood. As a result, complete resection of the tumour was achieved with minimal difficulty. A patch of pericardium was sutured with 5-0 Prolene to repair the defect. Following that, the mitral valve was excised and the St Jude Regent No. 21 tissue valve was implanted. Post-operative histopathology examination (HPE) of the mass revealed multiple thin- and thick-walled dilated vessels, suggesting a cavernous type of haemangioma (Fig. 4).

Post-operatively, patient was observed in the hospital for 2 weeks for another neurological illness and subsequently discharged well on January 24th, 2018. The chest tightness and SOB improved significantly after the operation, resulting in better exercise tolerance. After 3 months, the patient returned to the hospital for follow up. There were no new complaints. No haemangioma recurrence was seen on CTA.

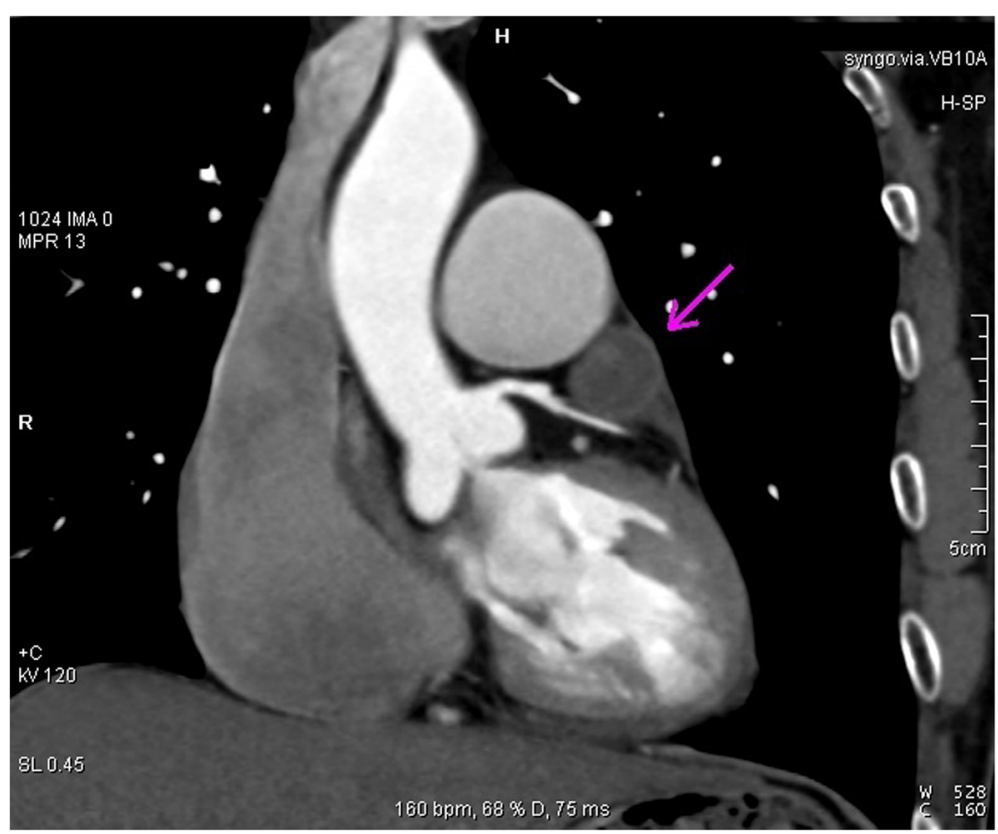

Fig. 1 Cardiac 2D CTA reconstruction. The purple arrow points to the mass 


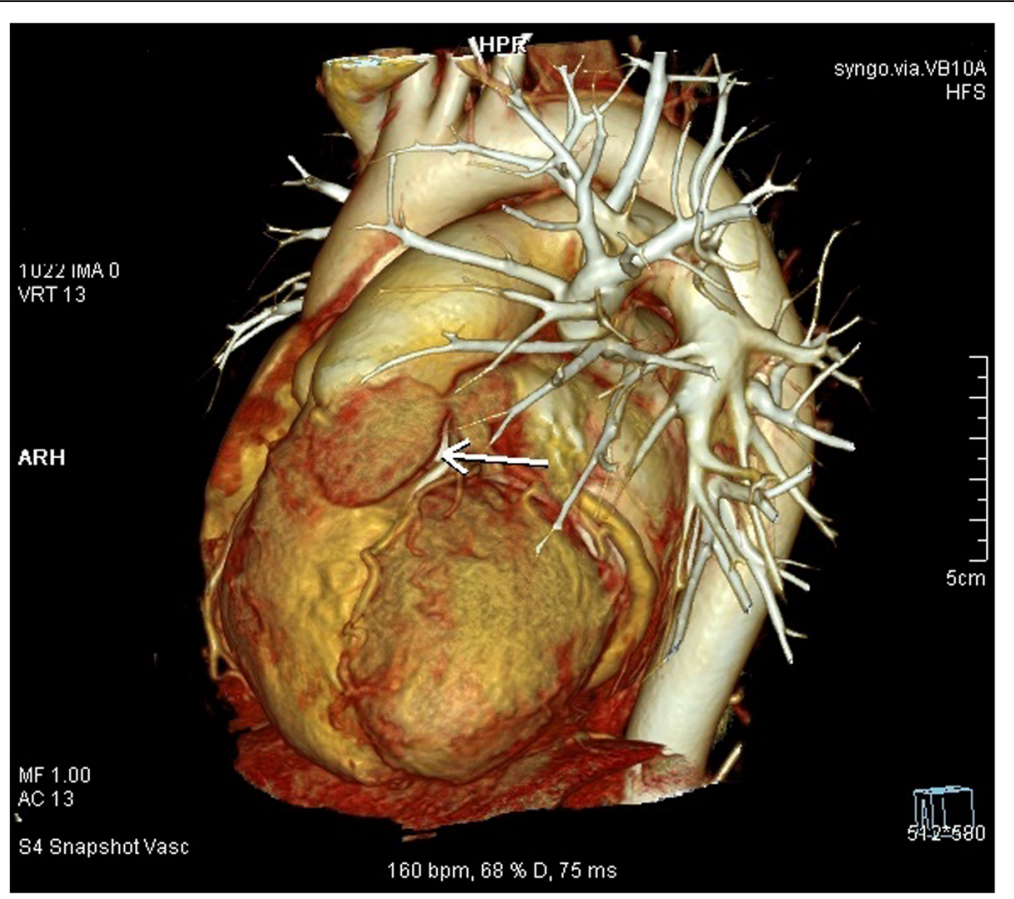

Fig. 2 Cardiac 3D CTA reconstruction. The white arrow points to the mass

\section{Discussion}

Cardiac haemangiomas share the same histological features with haemangiomas arising from other parts of the body. All haemangiomas are made up of benign proliferation of endothelial cells. Cardiac haemangiomas can originate from any layers of the heart or throughout the whole pericardium. Demographically, haemangiomas have been detected in almost all age group. However, there is a slight predominance among female patients. Some of the cardiac haemangiomas are asymptomatic and detected incidentally. However, depending on the

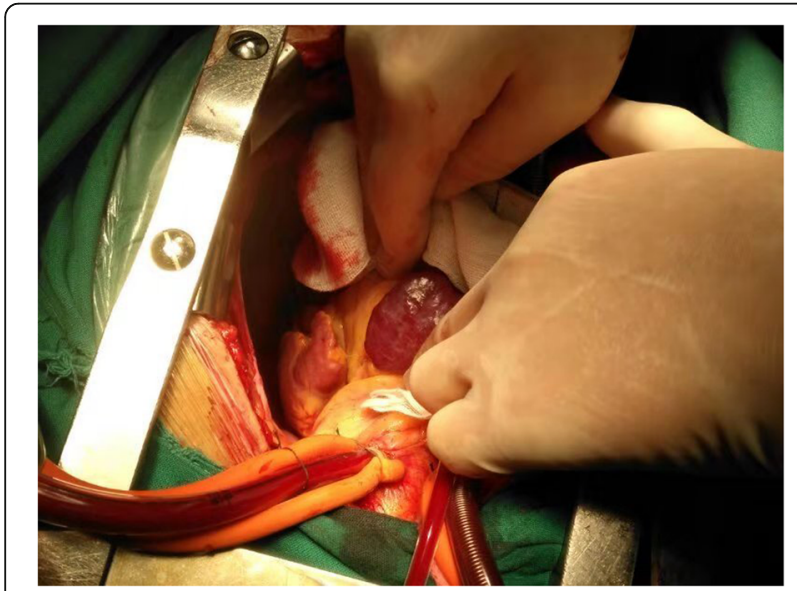

Fig. 3 Intraoperative view of the tumour. A heart-shaped mass, was visible on the surface of the anterior wall of the right ventricle. It was approximately $3 \times 2 \mathrm{~cm}$ in size

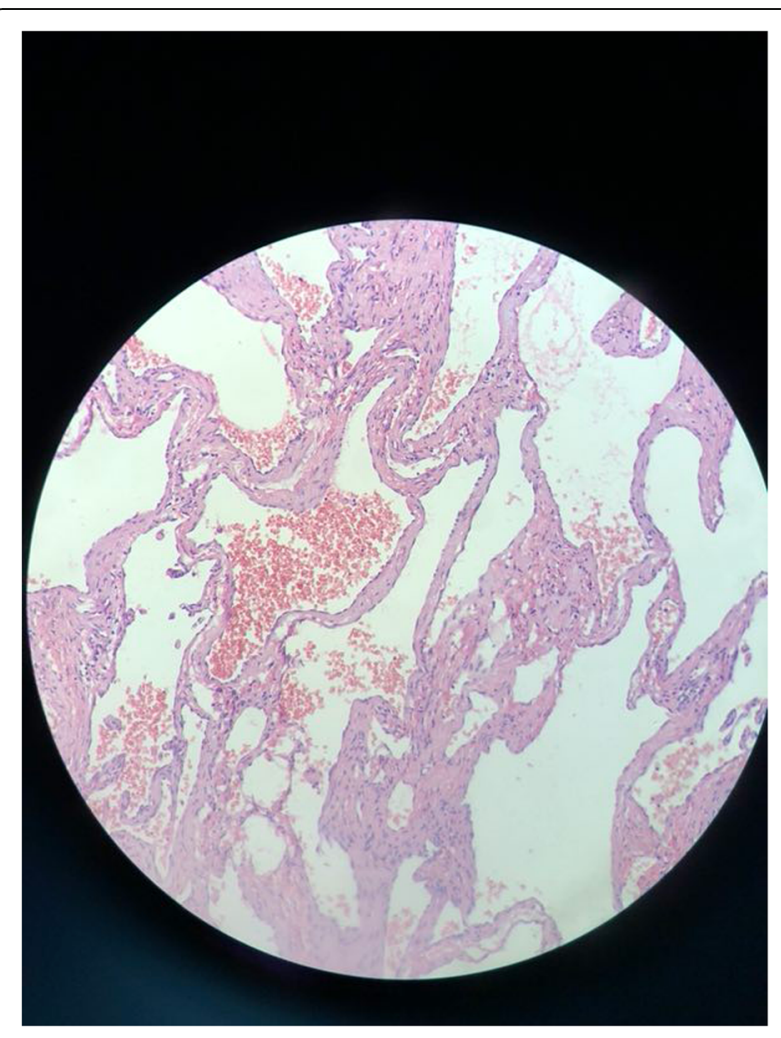

Fig. 4 Histopathology examination (HPE) of the mass revealed multiple thin- and thick-walled dilated vessels, confirming the diagnosis of cavernous haemangioma 
anatomic site of the tumour and the extension of the tumour margin, patients with cardiac haemangioma may manifest different types of symptoms. Some patients may complain of educed exercise tolerance, dyspnoea, palpitation, atypical chest pain, and irregular heartbeats.

Among the different types of cardiac haemangiomas, cavernous haemangiomas are associated with slow growth rate and low invasion rate of the adjacent structures. Nevertheless, as a result of external compression, cavernous haemangiomas may manifest clinically as outflow tract obstruction, atrial compression, ventricular dysrhythmias, and embolization in the patient [4].

Improvement in modern imaging modalities have greatly enhanced the diagnosis of cardiac haemangiomas. Traditionally, echocardiography is main investigation modality. Transoesophageal echocardiography is more useful than transthoracic echocardiography as it offers increased sensitivity in diagnosing cardiac haemangiomas [6]. However, echocardiography is invasive in nature and it also has certain limitations as it is not able to fully assess structures such as the mediastinum, diaphragm, and lung parenchyma that are distant to the transducer. A better modality would be $\mathrm{CT}$ as it is able to better demonstrate the tumour location, the relationship of the tumour with adjacent structures, and if there is any invasion into vital structures in the surrounding. Furthermore, CT may characterize the tumour lesion based on the attenuation values or pattern of enhancement in order to rule out other differential diagnoses. Another advantage of CT lies within its ability to determine if the tumour is confined regionally or it has metastasized to other distant structures.

In this patient, there was a rapid uptake of the iodinebased contrast by the highly vascular tumour during CT imaging. This proved to be useful in ruling out the intracardiac mass as a thrombus, as a thrombus would be non-enhancing in nature.

Nevertheless, magnetic resonance imaging (MRI) remains the gold standard of diagnosis of this condition as it provides a higher contrast resolution compared with CT. MRI is also able to detect any myocardial invasion apart from providing additional information about any functional effect the neoplasm might have incurred on the heart [7]. Last but not least, positron emission tomography (PET)/CT is another useful staging tool for cardiac haemangiomas as it can display any regional or distant spread of the tumours. However, its uptake is still low especially in financially constraint settings.

In this case, the patient was diagnosed as having a cardiac haemangioma. However, the site of origin of the tumour remained unclear. The natural history of cardiac cavernous haemangiomas is highly varied. With regard to the treatment modality, surgical excision is the preferred management for most surgeons. In view of the potential risk of embolism and rupture, it remains controversial to surgically remove all types of cardiac haemangiomas. However, it is almost universally accepted that symptomatic patients should undergo surgical resection. With adequate surgical resection, the long-term prognosis is often favourable. In contrast, patients who are undiagnosed and untreated may suffer from sudden death due to arrhythmias.

In short, cardiac haemangiomas are a type of rare but benign tumour which most commonly arise from the right side of the heart. These cardiac haemangiomas often have unpredictable natural histories and no specific clinical manifestations, resulting in diagnosis challenge. Despite so, imaging modality such as CT and MRI is a very useful in diagnosing and evaluating the surgical resectability of the haemangioma. Complete surgical excision remains the first line therapy. For majority of the patients, prognosis is generally favourable if detected and managed promptly.

\section{Abbreviations \\ CT scan: Computed tomography scan; CTA: Computed tomography angiography; HPE: Histopathology examination; MRI: Magnetic resonance imaging; PET: Positron emission tomography}

\section{Acknowledgements}

We would like to express our deep gratitude to Professor Zhao Lu Ning for his guidance and critiques for case report. We would also like to extend our gratitude to the all the radiology technicians in the Department of Cardiothoracic for the high-quality images in this case.

\section{Authors' contributions}

MYI wrote the manuscript. HJ collected all the relevant materials including the figures and medical records. XMB performed the surgical procedure. All authors have read and approved the manuscript.

Funding

None.

Availability of data and materials

All data can be found at the first affiliated hospital of Dalian medical university, cardiothoracic surgery department.

Ethics approval and consent to participate

Written informed consent was obtained from the patient to use this case and any accompanying images for the purpose of journal manuscript publication. A copy of the written consent is available for review by the journal. Ethical approval was not necessary as this is a case study.

\section{Consent for publication}

Written consent for publication was obtained from the patient in this case study

\section{Competing interests}

None.

Received: 11 April 2019 Accepted: 15 July 2019

Published online: 29 July 2019

References

1. Thomas JE, Eror AT, Kenney M, et al. Asymptomatic right atrial cavernous haemangioma:a ease report and review of the literature [J].Cardiovasc Pathol, 13(6):34l-344.DOl:https://doi.org/10.1016/j.carpath.2004.09.001. 
2. Jonjev ZS., Torbica V, Vuekovi6, et al. Cavernous haemangioma of the heart[J]. Herz, 2014, 39(6):716-717.DOl:https://doi.org/10.1007/s00059-013-3 854-7.

3. Burke A, Vermani R. Atlas of tumour pathology: tumours of the heart and great vessels. Silver Spring, MD: American Registry of Pathology; 1996.

4. Yusuf S, Reardon M, Banchs J. Cardiac tumours. Cardiology. 2014;129:197-8.

5. Li W, Teng P, Xu H, Ma L, Ni Y. Cardiac hemangioma: a comprehensive analysis of 200 cases. Ann Thorac Surg. 2015;99(6):2246-52

6. Lamba G, Frishman WH. Cardiac and pericardial tumors. Cardiol Rev. 2012; 20(5):237-52.

7. Randhawa K, Ganeshan A, Hoey ET. Magnetic resonance imaging of cardiac tumors: part 1, sequences, protocols, and benign tumors. Curr Probl Diagn Radiol. 2011;40(4):158-68

\section{Publisher's Note}

Springer Nature remains neutral with regard to jurisdictional claims in published maps and institutional affiliations.

Ready to submit your research? Choose BMC and benefit from:

- fast, convenient online submission

- thorough peer review by experienced researchers in your field

- rapid publication on acceptance

- support for research data, including large and complex data types

- gold Open Access which fosters wider collaboration and increased citations

- maximum visibility for your research: over $100 \mathrm{M}$ website views per year

At BMC, research is always in progress.

Learn more biomedcentral.com/submissions 